\title{
The Role of Cell Metabolism in Innate Immune Memory
}

\author{
Anaisa Valido Ferreira ${ }^{a, b} \quad$ Jorge Domiguéz-Andrés ${ }^{a} \quad$ Mihai Gheorghe Netea ${ }^{a, c, d}$ \\ aDepartment of Internal Medicine and Radboud Center for Infectious Diseases (RCI), Radboud University Nijmegen \\ Medical Center, Nijmegen, The Netherlands; ${ }^{b}$ Instituto de Ciências Biomédicas Abel Salazar (ICBAS), Universidade \\ do Porto, Porto, Portugal; ' Department for Genomics \& Immunoregulation, Life and Medical Sciences Institute

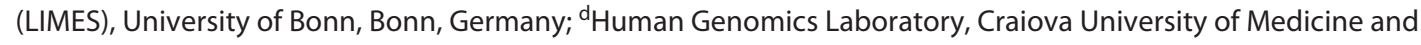 \\ Pharmacy, Craiova, Romania
}

\section{Keywords}

Metabolism · Trained immunity · Therapies

\begin{abstract}
Immunological memory is classically attributed to adaptive immune responses, but recent studies have shown that challenged innate immune cells can display long-term functional changes that increase nonspecific responsiveness to subsequent infections. This phenomenon, coined trained immunity or innate immune memory, is based on the epigenetic reprogramming and the rewiring of intracellular metabolic pathways. Here, we review the different metabolic pathways that are modulated in trained immunity. Glycolysis, oxidative phosphorylation, the tricarboxylic acid cycle, amino acid, and lipid metabolism are interplaying pathways that are crucial for the establishment of innate immune memory. Unraveling this metabolic wiring allows for a better understanding of innate immune contribution to health and disease. These insights may open avenues for the development of future therapies that aim to harness or dampen the power of the innate immune response.

(c) 2020 The Author(s).

Published by S. Karger AG, Basel
\end{abstract}

karger@karger.com www.karger.com/jin

Karger

GOPEN ACCESS
C 2020 The Author(s).

Published by S. Karger AG, Basel

This is an Open Access article licensed under the Creative Commons Attribution-NonCommercial-4.0 International License (CC BY-NC) (http://www.karger.com/Services/OpenAccessLicense), applicable to the online version of the article only. Usage and distribution for commercial purposes requires written permission.

\section{Introduction}

Immunological memory has been classically attributed solely to the adaptive immune system. Once activated, $\mathrm{B}$ and $\mathrm{T}$ cells proliferate and confer specific long-lasting memory and thus award protection. Although invertebrates and plants lack an adaptive immune system, it is well documented that they exhibit greater resistance to subsequent infections. Specifically, systemic acquired resistance has been described in plants, whereby the first immune response confers long-term protection against a wide range of pathogens [1]. In addition, epidemiological studies point that vaccines, notably in live-attenuated forms, such as measles, polio oral vaccine, or Bacillus Calmette-Guérin (BCG), elicit nonspecific effects that reduce mortality due to protection against non-targeted diseases $[2,3]$. Presently, there is a growing body of evidence in human and murine models that support the concept that the innate immune system also has features of long-term memory, whereby innate immune cells encounter an inflammatory challenge and later respond in a heightened manner to a secondary event. This phenomenon has been termed innate immune memory or trained immunity and does not rely on a particular stimulant,

Mihai Gheorghe Netea

Department of Internal Medicine and Radboud Center for Infectious Diseases (RCI) Radboud University Medical Center

Geert Grooteplein Zuid 8, NL-6525 GA Nijmegen (The Netherlands) mihai.netea@ radboudumc.nl 


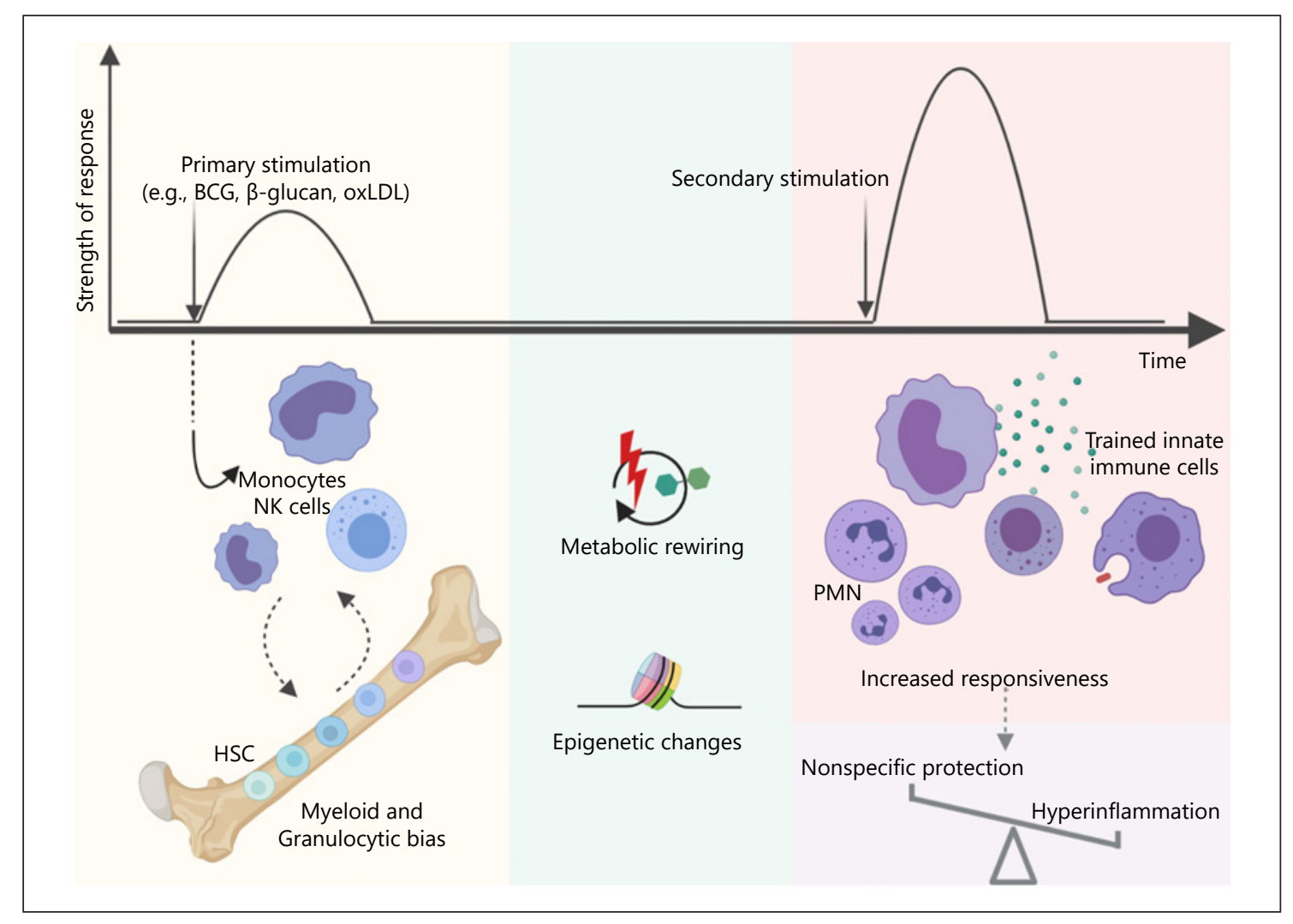

Fig. 1. Innate immune memory. Pathogens or non-microbial ligands activate the innate immune system and induce metabolic rewiring and epigenetic changes. This modulation also leads to a myeloid and granulocytic bias of the HSC compartment, promoting the production and release of trained innate immune cells, which exhibit increased responsiveness to posterior heterologous restimulation. Thus, trained immunity contributes to long-term nonspecific protection conferred by vaccines, while promoting hyperinflammatory phenotypes. BCG, Bacillus Calmette-Guérin; OxPHOS, oxidative phosphorylation; PMN, polymorphonuclear leukocytes; HSC, hematopoietic stem cell. Figure was created with Biorender.com. thus conferring protection against heterologous pathogens (Fig. 1) $[4,5]$. Mice inoculated with the fungus Candida albicans became resistant to a secondary lethal infection. This protection was still observed in Rag1-deficient mice, demonstrating that the phenomenon is independent of T-and B-cell function [6].

Innate immune memory has been described primarily in myeloid cells, such as monocytes, macrophages, and possibly dendritic and mast cells in response to a variety of challenges. Lymphoid cells such as natural killer cells or innate lymphoid cells have also been recently shown to display long-term memory characteristics [7-9]. Monocytes or macrophages exposed to C. albicans, or the fungal cell wall component $\beta$-glucan, show enhanced production of proinflammatory cytokines upon a unrelated secondary stimulation such as LPS or Mycobacterium tuberculosis[6]. Sterile endogenous stimuli can also induce trained immunity. Oxidized low-density lipoprotein (oxLDL) induces a long-lasting proinflammatory phenotype in monocytes, which has been associated with atherosclerosis and foam cell formation [10]. However, a long-lasting protection cannot be secured by short-lived cells. Recent mouse studies have shown that BCG and $\beta$-glucan skew hematopoietic stem cells (HSCs) toward myelopoiesis, generating trained monocytes and ultimately conferring partial protection against pulmonary tuberculosis and chemotherapy-induced myeloablation, respectively $[11,12]$. Also, HSCs harvested from mice injected with LPS led to a decrease of mortality of the transplanted mice to Pseudomonas aeruginosa. This protection was still conferred by re-transplantation of the LPS exposed HSCs into a secondary host [13]. This central impact of trained immunity at the level of bone marrow progenitors was also recently explored in a human BCG vaccination study, 
Fig. 2. Trained immunity is supported by a metabolic rewiring. Trained cells exhibit an upregulation of glycolysis, TCA, OxPHOS, glutaminolysis, and fatty acid metabolism. OxPHOS and glycolysis provide ATP, while TCA cycle intermediates modulate the function of different proteins. Succinate inhibits the degradation of the transcription factor hypoxia-inducible factor 1- $\alpha$, which in turn promotes the transcription of various proinflammatory and glycolytic genes. Succinate also inhibits the enzymatic activity of histone demethylase lysine-specific demethylase 5 (KDM5), while acetyl-CoA is an acetyl donor for histone acetyltransferases. This promotes epigenetic changes that facilitate the transcription of proinflammatory genes triggered by a posterior stimulation. NADH, nicotinamide adenine dinucleotide; me, methyl; ac, acetyl; TCA, tricarboxylic acid; OxPHOS, oxidative phosphorylation; ATP, adenosine triphosphate. Figure was created with Biorender.com.

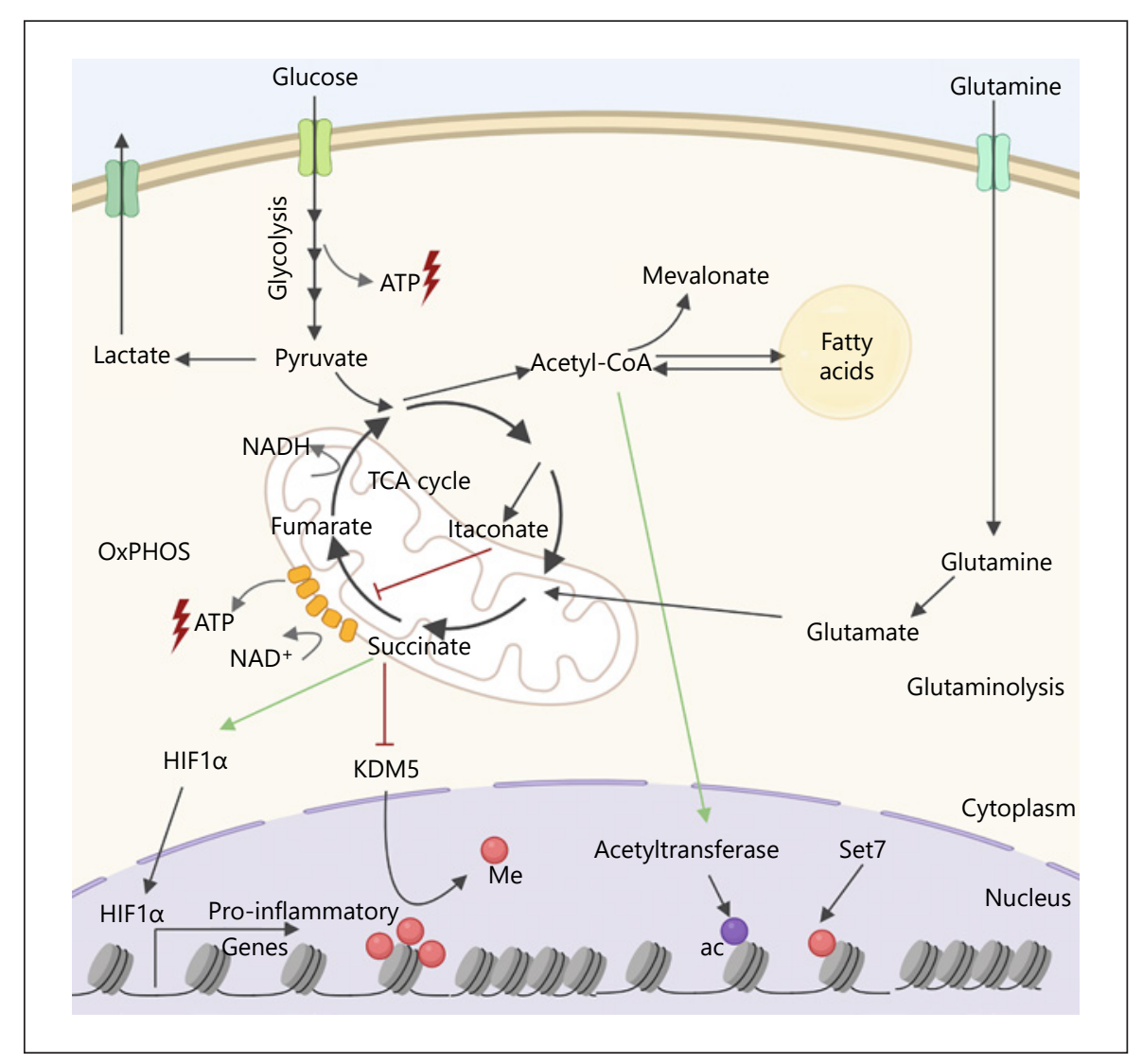

where it was demonstrated that the composition of HSCs did not change after vaccination. Nonetheless, BCG vaccination was shown to induce transcriptional changes in HSCs while concomitantly increasing responsiveness of innate immune cells to unrelated challenges [14]. In accordance, a cohort of BCG-vaccinated newborn infants exhibited an increase in neutrophil counts in comparison to non-vaccinated children [14]. In addition to HSCs, other stem cells may exhibit features of memory. Epithelial stem cells are progenitors of differentiated epithelial cells that form a physical barrier against external agents. Mouse models of skin sterile inflammation and fungal infection were used to show that epithelial stem cells persist after the skin returns to homeostasis and later contribute to enhanced epidermal wound healing of the previously inflamed skin [15].

Innate immune memory, in contrast to the adaptive immune memory, is not conferred neither by genomic rearrangements nor by clonal diversity and selection. Instead, the different inducers of trained immunity trigger epigenetic changes in the host cell. Trained cells harbor stable chromatin marks in gene regulatory regions that seemingly have no effect on steady-state gene expression.
These chromatin adaptations modulate its accessibility to the transcriptional machinery and confer a long term increased gene transcription in response to a secondary trigger. A second hallmark of trained cells is the rewiring of metabolic pathways. Cellular metabolism has not only been correlated to cell function, but also is an important regulator of epigenetic changes. Metabolites act as signaling molecules, cofactors, and substrates that modulate the activity of chromatin modifying enzymes. This close interplay between metabolism and epigenetic reprograming urges further exploration to understand the mechanisms underlying the different cellular metabolic pathways implicated in innate immune memory. The present review will discuss current findings that put metabolic pathways such as glycolysis, oxidative respiration, and the tricarboxylic acid (TCA) cycle at the center of the induction of trained immunity (Fig. 2). Furthermore, we will discuss future therapeutic strategies that may arise from the modulation of innate immune pathways aimed at the dampening of the trained phenotype present in proinflammatory diseases or at inducing the increased responsiveness in immunosuppressed conditions. 


\section{Immunometabolic Pathways Involved in Trained Immunity Glycolysis and Oxidative Respiration}

Cells are extremely plastic and can adjust their metabolism according to their bioenergetic needs that change to adapt to microenvironmental changes. Glucose breakdown rapidly produces adenosine triphosphate (ATP) and pyruvate. Pyruvate can then be used in the TCA cycle or be further oxidized to lactate. Glycolysis is often increased in activated $\mathrm{T}$ cells [16] and proinflammatory macrophages [17]. In accordance, trained macrophages have an increased glucose consumption and release high levels of the glycolysis end-product lactate [18]. This pathway is not an efficient ATP producer (only 2 ATP molecules are produced from one molecule of glucose) but can be quickly stimulated and amplified. The enhanced glycolytic rate is enabled by the increased expression of glycolytic enzymes which is sustained through epigenetic changes [19]. Glycolysis increase is common to different training stimuli such as $\beta$-glucan, BCG, and oxLDL and has been described not only in the monocytemacrophage populations but also in HSCs of $\beta$-glucanexposed mice [12]. This hallmark of trained immunity is mediated by the AKT-mTOR HIF1 a pathway $[19,20]$. In vitro inhibition of this pathway decreases the enhanced responsiveness of trained cells. Also, myeloid cell specific HIF1 $\alpha$ knockout mice exposed to $\beta$-glucan were more sensitive to an $S$. aureus infection than their trained wildtype controls [20,21]. In addition, humans treated with metformin, a known inhibitor of mTOR, presented decreased responsiveness to restimulation after BCG ex vivo training [19].

In contrast, oxidative respiration relies on the Krebs cycle and mitochondrial machinery to generate ATP, which occurs more efficiently (in total 36 molecules of ATP from 1 molecule of glucose) but at a slower rate than glycolysis. In OXPHOS, molecules of the electron donor nicotinamide adenine dinucleotide are oxidized and donate electrons to the electron transport chain. There, electrons are sequentially relayed through the ETC complexes resulting in the reduction of oxygen and the pumping of protons across the inner membrane, generating water and a proton gradient. The shuttled protons then drive ATP synthesis generated by F0F1-ATP synthase. A pioneering study first described that $\beta$-glucan-induced memory in human monocytes leads to a metabolic rewiring with an increase of aerobic glycolysis and a dampened OXPHOS pathway [20]. However, further studies with lower doses of $\beta$-glucan or with BCG as a inducer of innate immune memory show that, concomitantly to an in-

Cellular Metabolism and Innate Immune Memory crease in glycolysis, these cells consume higher levels of oxygen $[19,22,23]$. This highly energetic state, where glycolytic and OXPHOS pathways are both potentiated, was further observed in human monocytes exposed to the sterile innate immune memory inducer oxLDL [24]. In addition, a recent study has described suggestive associations between common single nucleotide polymorphisms in genes related to OXPHOS and variation in the degree of responsiveness of $\beta$-glucan trained monocytes [23]. These data collectively suggest that the metabolic shift toward aerobic glycolysis and the classically described Warburg effect is not common to the establishment of innate immune memory. Instead, enhanced OXPHOS appears as a regulator of the induction of the trained phenotype.

Interestingly, the enhanced electron-hungry OXPHOS pathway might contribute to the higher NAD $(\mathrm{P})+$ / $\mathrm{NAD}(\mathrm{P}) \mathrm{H}$ ratio observed in trained cells [20,21], which may influence mitochondrial metabolism. Mitochondrial ATP production relies on nicotinamide adenine dinucleotide, which is produced by the TCA cycle and fatty acid $\beta$-oxidation. Curiously, monocytes exposed to oxLDL exhibited increased mitochondrial ROS production, which was ablated upon mTOR inhibition [21]. These findings might suggest a relevant role for mitochondria function and metabolism in the induction of trained immunity that warrants further exploration.

\section{TCA Cycle and Amino Acid Metabolism}

In addition to providing for the energetic needs of the cell, metabolic rewiring also modulates the metabolic substrates available. Different metabolites have been attributed signaling functions by inhibiting the activity of enzymes or triggering protein post-translational modifications. Specifically, the TCA cycle integrates multiple catabolic and anabolic pathways. In short, glycolysis supplies pyruvate that is metabolized into acetyl-CoA, the fuel of the TCA cycle. Acetyl-CoA can also be derived from the $\beta$-oxidation of fatty acids, while the TCA cycle intermediate citrate can be used as a precursor for lipid biosynthesis. An alternative carbon source is provided by glutaminolysis, which replenishes the TCA cycle by the conversion of glutamate into $\alpha$-ketoglutarate $(\alpha-K G)$. Also, the oxidation of succinate to fumarate by the enzyme succinate dehydrogenase, known as complex II of the ETC, shuttles an electron to power ATP production by the OXPHOS pathway $[25,26]$.

The induction of innate immune memory hinges on the accumulation of TCA cycle intermediate metabolites, 
which may result from the interplay between glycolysis and glutaminolysis [18]. The transcription factor HIF1a, activated in trained cells, blocks the conversion of pyruvate to acetyl-CoA by inhibiting pyruvate dehydrogenase [27]. Conversely, glutamine metabolism is increased and supplements the TCA cycle with $\alpha$-KG derived from glutamate. Glutaminolysis inhibition impaired the prototypical increased responsiveness of $\beta$-glucan trained macrophages [18]. In addition to glutamine, trained cells show an enhanced consumption of the amino acid aspartate; however, its role in the induction of trained immunity remains to be clarified. Methionine consumption is also increased in $\beta$-glucan exposed monocytes [18]. It is intriguing to consider that methionine is the precursor of S-adenosylmethionine (SAM), a methyl donor critical for DNA and histone methylation which plays a central role in chromatic dynamics and gene expression patterns [28].

Metabolic rewiring is crucial for long-term gene expression and epigenetic changes in innate immune memory. Trained monocytes amass increased levels of succinate and fumarate, which are competitive inhibitors of multiple $\alpha-K G$ dependent dioxygenases ( $\alpha$-KGDD) [29]. The $\alpha-K G D D$ family of enzymes include histone and DNA demethylases such as the Jumonji (Jmj)-KDM5 family which actively remove lysine trimethylation, such as $\mathrm{H} 3 \mathrm{~K} 4 \mathrm{me} 3$ and $\mathrm{H} 3 \mathrm{~K} 27 \mathrm{me} 3$ marks [30]. The cell-permeable monomethyl fumarate was shown to increase the responsiveness of human monocytes, enrich the activating mark $\mathrm{H} 3 \mathrm{~K} 4 \mathrm{me} 3$ deposition at the promoter regions of the proinflammatory genes TNFA and IL6, while also inhibiting the activity of KDM5 [18]. Succinate and fumarate also have the relevant role of stabilizing HIF1 $\alpha$ by the inhibition of prolyl hydroxylases, which constitutively promote the degradation of HIF1a. The increased levels of the transcription factor HIF1 $\alpha$ also promote IL- $1 \beta$ transcription and upregulation of glycolysis [31]. HIF1a induces the expression of histone demethylases [32], adding another possible indirect way through which the ratios of succinate, fumarate, and $\alpha-K G$ could influence epigenome remodeling.

SDH, or complex II, oxidizes succinate into fumarate and shuttles an electron along the ETC. $\beta$-glucan trained monocytes show increased expression of different SDH subunits [33]. Also, genetic human studies have identified suggestive SNPs in SDH subunit coding genes associated with changes in responsiveness $[23,33]$. The regulation of transcription of SDH genes has been shown to be modulated by the lysine methyltransferase Set7. In vitro pharmacological inhibition of Set7 or the use of SETD7 knockout mice led to a decrease of the $\beta$-glucan-induced upregulation of SDHB transcripts [23]. Interestingly, another modulator of SDH activity also regulates the trained immunity phenotype: itaconate is derived from the TCA cycle and produced by macrophages in proinflammatory conditions [34]. Considered as a break mechanism to the proinflammatory response, itaconate is attributed antiinflammatory and antioxidant properties. It activates the master regulator of the antioxidant response, the transcription factor NRF2 [35], and is an inhibitor of SDH by occupying the binding pocket of succinate [36]. Notably, the in vitro $\beta$-glucan trained phenotype is hindered by concomitant exposure to the membrane permeable derivative dimethyl itaconate, possibly due to $\mathrm{SDH}$ inhibition [33]. This break in the TCA cycle could in turn compromise the fumarate accumulation necessary for epigenetic remodeling. Itaconate has also been described to alkylate thiol groups and interact with the ROS scavenger glutathione [37], the glycolytic enzymes lactate dehydrogenase [35], glyceraldehyde 3-phosphate dehydrogenase (GAPDH) [38], and fructose-bisphosphate aldolase [39]. Interestingly, lactate dehydrogenase and GAPDH are also possible targets for succinylation [31]. However, whether such mechanisms play a role in innate immune memory remains to be elucidated. The discovery of itaconate as a relevant immunomodulator in the context of mammalian responses is recent $[34,40,41]$, and exciting new studies are exploring the intricate role of this metabolite in diverse inflammatory contexts [42-44].

\section{Lipid Metabolism}

Cells obtain fatty acids from de novo lipogenesis and exogenous uptake. Glycolysis, glutaminolysis, and the TCA cycle contribute to produce citrate, which in turn is converted to acetyl-CoA and gives rise to palmitate. Palmitate can be further elongated and desaturated to generate a multitude of lipid species, which can be subsequently stored as lipid droplets. These organelles contribute to the production of inflammatory mediators that regulate immune responses [45]. The stored fatty acids can be degraded to restore acetyl-CoA levels through $\beta$-oxidation. Interestingly, in vitro exogenous supplementation of acetyl-CoA induced a proinflammatory phenotype in human monocytes [46]. Acetyl-CoA serves as an acetyl donor that may favor histone acetylation and fuels the cholesterol pathway and fatty acid biosynthesis. Mevalonate, a metabolite of the cholesterol pathway, has been shown to regulate trained immunity [47]. Indeed, statins, which inhib- 
it the rate-limiting enzyme that produces mevalonate, decreased the responsiveness induced by $\beta$-glucan, BCG, and oxLDL [47]. Moreover, mutations in mevalonate kinase, which lead to mevalonate accumulation, trigger an autoinflammatory clinical phenotype, the hyper immunoglobulin $\mathrm{D}$ syndrome. These patients show a constitutive trained immunity profile which could contribute to their clinical picture of sterile inflammation attacks [47]. Central trained immunity also relies on the mevalonate pathway as shown by the accumulation of various species of cholesterol esters in HSCs. Inhibition of the enzyme upstream of mevalonate in a mouse model of $\beta$-glucan induced innate immune memory reduced HSC expansion bias toward myelopoiesis [12]. Interestingly, systemic increase of cholesterol and inflammatory mediators also triggered innate immune memory in an atherosclerosis mouse model. Monocytic cells isolated from mice exposed to high-fat diet for a limited period showed increased responsiveness to ex vivo stimulation, even after the animals returned to a chow diet. Concomitantly the hematopoietic cell compartment proliferated and was biased toward granulocyte monocyte precursor cells, in an NLRP3 inflammasome-dependent manner [48].

Fatty acid synthesis also appears to play a role in the induction of innate immune memory. Hematopoietic progenitors of $\beta$-glucan-trained mice were enriched for lipids with shorter and more saturated acyl chains with a decrease of alkyl-ether-linked phosphatidylcholines and phosphatidylethanolamines [12]. In accordance, HSC of mice trained with LPS and exposed to a second injection of LPS exhibited increased expression of genes related to fatty acid metabolism and oxidative phosphorylation [13]. The hormone aldosterone has also been recently described to induce trained immunity in vitro and epigenetically regulate the promoter regions of genes of the fatty acid synthesis. Moreover, the increased innate immune responsiveness induced by aldosterone was ablated by inhibition of this pathway [49].

The nuclear liver X receptor (LXR) is a transcription factor that coordinates gene expression linked to cholesterol and fatty acid metabolism. Pharmacological inhibition of LXR decreased the responsiveness of BCG-trained macrophages [46]. Moreover, LXR activation alone could replicate the macrophage trained phenotype, which was also dependent on the mevalonate pathway. Other transcription factors such as sterol regulatory element binding protein and peroxisome proliferator-activated receptors are regulators of lipid metabolism; however, their involvement in the induction of innate immune memory warrants further exploration [50].

Cellular Metabolism and Innate Immune Memory

\section{Perspectives for Therapies}

Trained immunity is considered an evolutionary adaptation that allows for long-lasting broad-spectrum resistance to pathogens, increasing the host's capacity to mount an immunological response. Conversely, this responsive immune state can become maladaptive and exacerbate chronic inflammatory conditions if inappropriately activated by endogenous ligands. Characterizing the specific changes that distinct innate immune cells subsets undergo in different diseases, dependent on stimuli and microenvironments, could aid in enhancing or curbing the effects of trained immunity.

Inhibition of innate immune memory could be beneficial for inflammatory conditions found in atherosclerosis [10], organ transplants [51], central nervous system inflammation [52], lupus erythematous [53], and chronic diseases associated with the Western-type lifestyle $[48,54]$. On the other hand, the enhanced responsiveness of trained cells can be harnessed to improve an impaired host defense, such as in patients suffering from immunodeficiencies, immunoparalysis [55, 56], immunosenescence [57], or cancer [12]. Tumorassociated macrophages are often immunosuppressive and allow tumor progression. In this line, BCG is often used as an immunotherapy in the treatment of bladder cancer [58]. Although immune system activation allows for elimination of cancer cells, chronic inflammation may also promote neoplastic transformation and tumor progression [59]. Consequently, it is of importance to explore therapies that induce trained immunity in a durable manner but that can also be inhibited once clinical benefit is achieved. Neurodegenerative diseases are associated with chronic inflammation and may be exacerbated by peripheral infections, and possibly by trained immunity. Innate immune memory triggered on a genetic mouse model of Alzheimer by LPS led to an increase of amyloid plaque formation and associated neuritic damage [60]. Also associated with age is immunosenescence, the gradual deterioration of the immune system [61]. Therapies that combine the induction of adaptive and innate immune memory could ameliorate immunosenescence. In this regard, BCG vaccination decreased the incidence of new respiratory infections in elderly individuals in a recent randomized clinical trial [62]. At the same time, BCG vaccination administration prior to other vaccines was able to boost antibody responses $[63,64]$. In addition, BCG vaccination has been hypothesized as a preventive measure against the newly emerged SARS-CoV2 virus infection and multi-

J Innate Immun 2022;14:42-50

DOI: 10.1159/000512280 
ple randomized controlled trial are currently testing this potential [65].

Innate immune memory could be inhibited or potentiated at different levels, namely by engaging specific receptors or modulating cellular metabolism and epigenome. Fungal ligands rely on the dectin-1 pathway [66], the metabolite mevalonate signals through the insulin growth factor 1 receptor [47], and $\mathrm{N}$-acetyl muramic acid has been shown to induce an NOD2-dependent trained immunity phenotype [67]. Also, clinical trials have shown the therapeutic value of anti-IL- $1 \beta$ for rheumatoid arthritis, gout, type 2 diabetes and cardiovascular disease [68, 69], possibly through the inhibition of trained immunity mechanisms. Inhibition of glycolysis and mTOR as well as the mevalonate pathway could contribute to a decrease in trained immunity, as is seen in vitro with the use of rapamycin $[18,20]$ and fluvastatin [47], respectively. However, epigenetic rewiring ensures the longevity of innate immune memory leading to persistence disease. As such, modulating the epigenetic landscape with inhibition of histone or DNA methyltransferases is an attractive alternative [70]. As mentioned above, different inhibitors of innate immune memory pathways have been tested in vitro, but in vivo usage encompasses new challenges. Adequate targeting to myeloid and HSCs is crucial as it decreases off-target effects and enhances bioavailability while decreasing toxicity. The use of functionalized nanoparticles could aid in targeting and in the delivery of desired compounds. In a mouse model of heart transplant, the trained phenotype of the infiltrating cells was reduced by administration of a nanoparticle that targeted myeloid cells for inhibition of mTOR [51]. In addition to pharmacological modulators, the recently developed CRISPR/dCas9 could also be exploited in the future. dCas 9 blocks transcription of a targeted gene by decreasing its accessibility or can also be modified to facilitate the recruitment of transcriptional activators and thus promote the transcription of a specific gene [71]. This technology is currently under development, but it might prove a viable therapeutic option in the future, for gene expression regulation in general, and specifically in the field of innate immune responses.

\section{Conclusion}

Innate immune memory relies on several metabolic pathways that provide the metabolites and energy necessary for epigenetic changes. Glycolysis, oxidative phosphorylation, glutaminolysis, the TCA cycle, and choles- terol metabolism have been shown to be at the center of this enhanced responsiveness. Other mechanisms, such as mitochondrial respiration, redox, and lipid metabolism may be possible players in the induction of trained immunity and warrant future exploration. However, further technological advancements in the field of metabolomics and epigenetics are necessary to promote the identification of further relevant pathways and the interplay between them. Ultimately, innate immune memory has been attributed a contributing role for the pathogenesis of autoinflammatory and autoimmune diseases. In contrast, an impaired innate immune system might be stimulated by inducers of trained immunity and regain responsiveness. This dichotomy makes trained immunity an attractive therapeutic target. However, the development of future effective and safe therapies will first call for an increased effort toward thorough understanding of not only the molecular mechanisms regulating innate immunity but also of the contribution of non-immune and microenvironmental players.

\section{Conflict of Interest Statement}

M.G.N. is a scientific founder of TTxD.

\section{Funding Sources}

A.V.F. is supported by a Fundação para a Ciência e a Tecnologia (FCT) PhD Grant PD/BD/135449/2017. M.G.N. is supported by an ERC Advanced Grant (\#833247) and by a Spinoza Grant of the Netherlands Organization for Scientific Research.

\section{Author Contributions}

A.V.F. wrote the first draft and J.D.A. and M.G.N. edited and reviewed it.

References

1 Netea MG, Van Der Meer JWM. Commentary trained immunity: an ancient way of remembering. Cell Host Microbe. 2017;21(3): 297-300.

2 Benn CS, Netea MG, Selin LK, Aaby P. A small jab: a big effect: nonspecific immunomodulation by vaccines. Trends Immunol. 2013;34(9):431-9.

3 Andersen A, Fisker AB, Rodrigues A, Martins C, Ravn H, Lund N, et al. National immunization campaigns with oral polio vaccine reduce all-cause mortality: a natural experiment within seven randomized trials. Front Public Health. 2018 Feb 2;6(13):13. 
4 Netea MG, Joosten LA, Latz E, Mills KH, Natoli G, Stunnenberg HG, et al. Trained immunity: a program of innate immune memory in health and disease. Science. 2016 Apr 22; 352(6284):aaf1098.

5 Netea MG, Domínguez-Andrés J, Barreiro LB, Chavakis T, Divangahi M, Fuchs E, et al. Defining trained immunity and its role in health and disease. Nat Rev Immunol. 2020 Jun;20(6):375-88.

6 Quintin J, Saeed S, Martens JHA, Giamarellos-Bourboulis EJ, Ifrim DC, Logie C, et al. Candida albicans infection affords protection against reinfection via functional reprogramming of monocytes. Cell Host Microbe. 2012 Aug 16;12(2):223-32.

7 Sun JC, Beilke JN, Lanier LL. Adaptive immune features of natural killer cells. Nature. 2009 Jan 29;457(7229):557-61.

8 Martinez-Gonzalez I, Mathä L, Steer CA, Ghaedi M, Poon GF, Takei F. Allergen-experienced group 2 innate lymphoid cells acquire memory-like properties and enhance allergic lung inflammation. Immunity. 2016 Jul 19; 45(1):198-208.

9 Weizman OE, Song E, Adams NM, Hildreth AD, Riggan L, Krishna C, et al. Mouse cytomegalovirus-experienced ILC1s acquire a memory response dependent on the viral glycoprotein m12. Nat Immunol. 2019 Aug 1; 20(8):1004-11.

10 Siroon B, Jessica Q, Joosten Leo AB, van der Meer Jos WMNMG, Riksen Niels P. Oxidized low-density lipoprotein induces long-term proinflammatory cytokine production and foam cell formation via epigenetic reprogramming of monocytes. Arterioscler Thromb Vasc Biol. 2014 Aug;34(8):1731-8.

11 Kaufmann E, Sanz J, Dunn JL, Khan N, Mendonça LE, Pacis A, et al. BCG educates hematopoietic stem cells to generate protective innate immunity against tuberculosis. Cell. 2018 Jan 11;172(1-2):176-e19.

12 Mitroulis I, Ruppova K, Wang B, Chen LS, Grzybek M, Grinenko T, et al. Modulation of myelopoiesis progenitors is an integral component of trained immunity. Cell. 2018; 172(1-2):147-e12.

13 Kandalla PK, Amit I, Sarrazin S, Sieweke MH. $\mathrm{C} / \mathrm{EBP} \beta$-dependent epigenetic memory induces trained immunity in hematopoietic stem cells. Cell Stem Cell. 2020;26(5):657-74. e8.

14 Cirovic B, de Bree LCJ, Groh L, Blok BA, Chan J, van der Velden WJFM, et al. BCG vaccination in humans elicits trained immunity via the hematopoietic progenitor compartment. Cell Host Microbe. 2020;28(2):322-34. e5.

15 Naik S, Larsen SB, Gomez NC, Alaverdyan K, Sendoel A, Yuan S, et al. Inflammatory memory sensitizes skin epithelial stem cells to tissue damage. Nature. 2017 Oct 26;550(7677): $475-80$.
16 Menk AV, Scharping NE, Moreci RS, Zeng X, Guy C, Salvatore S, et al. Early TCR signaling induces rapid aerobic glycolysis enabling distinct acute $\mathrm{T}$ cell effector functions. Cell Rep. 2018 Feb 6;22(6):1509-21.

17 Viola A, Munari F, Sánchez-Rodríguez R, Scolaro T, Castegna A. The metabolic signature of macrophage responses. Front Immunol. 2019 Jul 3;10:1462.

18 Arts RJ, Novakovic B, ter Horst R, Carvalho A, Bekkering S, Lachmandas E, et al. Glutaminolysis and fumarate accumulation integrate immunometabolic and epigenetic programs in trained immunity. Cell Metab. 2016;24(6): 807-19.

19 Arts RJW, Carvalho A, La Rocca C, Palma C, Rodrigues F, Silvestre R, et al. Immunometabolic pathways in BCG-induced trained immunity. Cell Rep. 2016;17(10):2562-71.

20 Cheng SC, Quintin J, Cramer RA, Shepardson KM, Saeed S, Kumar V, et al. mTOR- and HIF-1 $\alpha$-mediated aerobic glycolysis as metabolic basis for trained immunity. Science. 2014;345(80-6204):1250684.

21 Sohrabi Y, Lagache SMM, Schnack L, Godfrey R, Kahles F, Bruemmer D, et al. mTOR-dependent oxidative stress regulates oxLDL-induced trained innate immunity in human monocytes. Front Immunol. 2018 Jan 22;9: 3155 .

22 Tarancó R, Domínguez-André J, Uranga S, Ferreira AV, Groh LA, et al. New live attenuated tuberculosis vaccine MTBVAC induces trained immunity and confers protection against experimental lethal pneumonia. PLoS Pathog. 2020 Apr 2;16(4):e1008404.

23 Keating S, Groh L, van der Heijden C, Rodriguez H, Dos Santos J, Fanucchi S, et al. The set7 lysine methyltransferase regulates plasticity in oxidative phosphorylation necessary for trained immunity induced by beta-glucan. Cell Rep. 2020 Apr 21;31(3):107548.

24 Keating ST, Groh L, Thiem K, Bekkering S, Li $\mathrm{Y}$, Matzaraki V, et al. Rewiring of glucose metabolism defines trained immunity induced by oxidized low-density lipoprotein. J Mol Med. 2020 Jun;98(6):819-31.

25 Martínez-Reyes I, Chandel NS. Mitochondrial TCA cycle metabolites control physiology and disease [Internet]. Nat Commun Nat Res. 2020;11:1-11

26 Ryan DG, O’Neill LAJ. Krebs cycle reborn in macrophage immunometabolism. Annu Rev Immunol. 2020 Apr 26;38:289-313.

27 Kim JW, Tchernyshyov I, Semenza GL, Dang CV. HIF-1-mediated expression of pyruvate dehydrogenase kinase: a metabolic switch required for cellular adaptation to hypoxia. Cell Metab. 2006 Mar 1;3(3):177-85.

28 Mentch SJ, Locasale JW. One-carbon metabolism and epigenetics: understanding the specificity. Ann N Y Acad Sci. 2016 Jan 1; 1363(1):91-8.
29 Xiao M, Yang H, Xu W, Ma S, Lin H, Zhu H, et al. Inhibition of $\alpha$-KG-dependent histone and DNA demethylases by fumarate and succinate that are accumulated in mutations of FH and SDH tumor suppressors. Genes Dev. 2012 Jun 15;26(12):1326-38

30 Cloos PAC, Christensen J, Agger K, Helin K. Erasing the methyl mark: histone demethylases at the center of cellular differentiation and disease [Internet]. Genes Dev. 2008 May 1;22(9):1115-40.

31 Tannahill GM, Curtis AM, Adamik J, Palsson-Mcdermott EM, McGettrick AF, Goel G, et al. Succinate is an inflammatory signal that induces IL-1 $\beta$ through HIF-1 $\alpha$. Nature. 2013 Apr 11;496(7444):238-42.

32 Batie M, Frost J, Frost M, Wilson JW, Schofield P, Rocha S. Hypoxia induces rapid changes to histone methylation and reprograms chromatin. Science. 2019 Mar 15; 363(6432):1222-6.

33 Domínguez-André J, Novakovic B, Li Y, Kumar V, Stunnenberg H, Netea Correspondence MG. The itaconate pathway is a central regulatory node linking innate immune tolerance and trained immunity. Cell Metab. 2019; 29:211-20.e5.

34 Michelucci A, Cordes T, Ghelfi J, Pailot A, Reiling N, Goldmann O, et al. Immune-responsive gene 1 protein links metabolism to immunity by catalyzing itaconic acid production. Proc Natl Acad Sci U S A. 2013 May 7; 110(19):7820-5.

35 Mills EL, Ryan DG, Prag HA, Dikovskaya D, Menon D, Zaslona Z, et al. Itaconate is an anti-inflammatory metabolite that activates Nrf2 via alkylation of KEAP1. Nature. 2018; 556(7699):113-7.

36 Cordes T, Wallace M, Michelucci A, Divakaruni AS, Sapcariu SC, Sousa C, et al. Immunoresponsive gene 1 and itaconate inhibit succinate dehydrogenase to modulate intracellular succinate levels. J Biol Chem. 2016; 291(27):14274-84.

37 Bambouskova M, Gorvel L, Lampropoulou V, Sergushichev A, Loginicheva E, Johnson K, et al. Electrophilic properties of itaconate and derivatives regulate the I $\mathrm{\kappa B} \zeta$-ATF3 inflammatory axis. Nature. 2018;556(7702):501-4.

38 Liao ST, Han C, Xu DQ, Fu XW, Wang JS, Kong LY. 4-Octyl itaconate inhibits aerobic glycolysis by targeting GAPDH to exert antiinflammatory effects. Nat Commun. 2019 Nov 8;10(1):5091.

39 Qin W, Qin K, Zhang Y, Jia W, Chen Y, Cheng $\mathrm{B}$, et al. S-glycosylation-based cysteine profiling reveals regulation of glycolysis by itaconate. Nat Chem Biol. 2019 Jul 22;15(10):983.

40 Shin JH, Yang JY, Jeon BY, Yoon YJ, Cho SN, Kang YH, et al. (1)H NMR-based metabolomic profiling in mice infected with Mycobacterium tuberculosis. J Proteome Res. 2011; 10(5):2238-47.

41 Sugimoto M, Sakagami H, Yokote Y, Onuma H, Kaneko M, Mori M, et al. Non-targeted metabolite profiling in activated macrophage secretion. Metabolomics. 2012;8(4):624-33. 
42 Riquelme SA, Liimatta K, Wong Fok Lung T, Fields B, Ahn D, Chen D, et al. Pseudomonas aeruginosa utilizes host-derived itaconate to redirect its metabolism to promote biofilm formation. Cell Metab. 2020 Jun 2;31(6): 1091-106.e6.

43 Daniels BP, Kofman SB, Smith JR, Norris GT, Snyder AG, Kolb JP, et al. The Nucleotide Sensor ZBP1 and Kinase RIPK3 Induce the Enzyme IRG1 to Promote an Antiviral Metabolic State in Neurons. Immunity. 2019 Jan; $0(0)$.

44 Zhao C, Jiang P, He Z, Yuan X, Guo J, Li Y, et al. Dimethyl itaconate protects against lippolysacchride-induced mastitis in mice by activating MAPKs and Nrf2 and inhibiting NF$\kappa B$ signaling pathways. Microb Pathog. 2019 Aug; 133:103541.

45 den Brok MH, Raaijmakers TK, ColladoCamps E, Adema GJ. Lipid droplets as immune modulators in myeloid cells. Trends Immunol. 2018;39(5):380-92.

46 Hermann Fritz J, Li Y, Sohrabi Y, De Hannes YS, Findeisen M, Sonntag GVH, et al. LXR activation induces a proinflammatory trained innate immunity-phenotype in human monocytes. Front Immunol. 2020 Mar 10;11: 353.

47 Bekkering S, Arts RJW, Novakovic B, Kourtzelis I, van der Heijden CDCC, Li Y, et al. Metabolic induction of trained immunity through the mevalonate pathway. Cell. 2018;172(1-2): 135-e9.

48 Christ A, Günther P, Lauterbach MAR, Duewell P, Biswas D, Pelka K, et al. Western diet triggers NLRP3-dependent innate immune reprogramming. Cell. 2018 Jan 11; 172(1-2):162-e14.

49 Van Der Heijden CDCC, Keating ST, Groh L, Joosten LAB, Netea MG, Riksen NP. Aldosterone induces trained immunity: the role of fatty acid synthesis. Cardiovasc Res. $2020 \mathrm{Feb}$ $1 ; 116(2): 317-28$.

50 Lachmandas E, Boutens L, Ratter JM, Hijmans A, Hooiveld GJ, Joosten LA, et al. Microbial stimulation of different Toll-like receptor signalling pathways induces diverse metabolic programmes in human monocytes. Nat Microbiol. 2017 Mar 19;2(3):16246.

51 Braza MS, van Leent MMT, Lameijer M, Sanchez-Gaytan BL, Arts RJW, Pérez-Medina C, et al. Inhibiting inflammation with myeloid cell-specific nanobiologics promotes organ transplant acceptance. Immunity. 2018 Nov 20;49(5):819-28.e6.
52 Wendeln AC, Degenhardt K, Kaurani L, Gertig M, Ulas T, Jain G, et al. Innate immune memory in the brain shapes neurological disease hallmarks. Nature. 2018 Apr 19; 556(7701):332-8.

53 Grigoriou M, Banos A, Filia A, Pavlidis P, Giannouli S, Karali V, et al. Transcriptome reprogramming and myeloid skewing in haematopoietic stem and progenitor cells in systemic lupus erythematosus. Ann Rheum Dis. 2020 Nov 28;79(2):242-53.

54 Bekkering S, van den Munckhof I, Nielen T, Lamfers E, Dinarello C, Rutten J, et al. Innate immune cell activation and epigenetic remodeling in symptomatic and asymptomatic atherosclerosis in humans in vivo. Atherosclerosis. 2016 Nov 1;254:228-36.

55 Novakovic B, Habibi E, Wang SY, Arts RJ, Davar R, Megchelenbrink W, et al. $\beta$-Glucan reverses the epigenetic state of LPS-induced immunological tolerance. Cell. 2016;167(5): 1354-68.e14.

56 Leentjens J, Kox M, Koch RM, Preijers F, Joosten LA, Van Der Hoeven JG, et al. Reversal of immunoparalysis in humans in vivo: a double-blind, placebo-controlled, randomized pilot study. Am J Respir Crit Care Med. 2012 Nov 1;186(9):838-45.

57 Bulut O, Kilic G, Domínguez-Andrés J, Netea MG. Overcoming immune dysfunction in the elderly: trained immunity as a novel approach. Int Immunol. 2020 Aug 7:dxaa052.

58 van Puffelen JH, Keating ST, Oosterwijk E, van der Heijden AG, Netea MG, Joosten LAB, et al. Trained immunity as a molecular mechanism for BCG immunotherapy in bladder cancer. Nat Rev Urol. 2020;17(9):513-25.

59 van Puffelen JH, Keating ST, Oosterwijk E, van der Heijden AG, Netea MG, Joosten LAB, et al. Trained immunity as a molecular mechanism for BCG immunotherapy in bladder cancer. Nat Rev Urol. 2020 Sep;17(9):513-25.

60 Wendeln AC, Degenhardt K, Kaurani L, Gertig M, Ulas T, Jain G, et al. Innate immune memory in the brain shapes neurological disease hallmarks. Nature. 2018 Apr 19; 556(7701):332-8.

61 Aiello A, Farzaneh F, Candore G, Caruso C, Davinelli S, Gambino CM, et al. Immunosenescence and its hallmarks: how to oppose aging strategically? A review of potential options for therapeutic intervention [Internet]. Front Immunol. 2019;10;2247.
62 Giamarellos-Bourboulis EJ, Tsilika M, Moorlag S, Antonakos N, Kotsaki A, DomínguezAndrés J, et al. Activate: randomized clinical trial of BCG vaccination against infection in the elderly. Cell. 2020 Oct 15;182(2):315-23. e9.

63 Leentjens J, Kox M, Stokman R, Gerretsen J, Diavatopoulos DA, Van Crevel R, et al. BCG vaccination enhances the immunogenicity of subsequent influenza vaccination in healthy volunteers: a randomized, placebo-controlled pilot study. J Infect Dis. 2015 Dec 15;212(12): 1930-8.

64 Arts RJW, Moorlag SJCFM, Novakovic B, Li $\mathrm{Y}$, Wang SY, Oosting $\mathrm{M}$, et al. BCG vaccination protects against experimental viral infection in humans through the induction of cytokines associated with trained immunity. Cell Host Microbe. 2018;23(1):89-e5.

65 O’Neill LAJ, Netea MG. BCG-induced trained immunity: can it offer protection against COVID-19? [Internet]. Nat Rev Immunol. 2020; 20:335-7.

66 Quintin J, Saeed S, Martens JHA, Giamarellos-Bourboulis EJ, Ifrim DC, Logie C, et al. Candida albicans infection affords protection against reinfection via functional reprogramming of monocytes. Cell Host Microbe. 2012 Aug 16;12(2):223-32.

67 Kleinnijenhuis J, Quintin J, Preijers F, Joosten LA, Ifrim DC, Saeed S, et al. Bacille CalmetteGuerin induces NOD2-dependent nonspecific protection from reinfection via epigenetic reprogramming of monocytes. Proc Natl Acad Sci U S A. 2012 Oct 23;109(43):1753742.

68 Dinarello CA, Simon A, Van Der Meer JW. Treating inflammation by blocking interleukin-1 in a broad spectrum of diseases. Nat Rev Drug Discov. 2012;11(8):633-52.

69 Baylis RA, Gomez D, Mallat Z, Pasterkamp G, Owens GK. The CANTOS trial: one important step for clinical cardiology but a giant leap for vascular biology. Arterioscler Thromb Vasc Biol. 2017;37(11):e174-7.

70 Tough DF, Tak PP, Tarakhovsky A, Prinjha RK. Epigenetic drug discovery: breaking through the immune barrier [Internet]. Nat Rev Drug Discov. 2016;15;835-53.

71 Maeder ML, Linder SJ, Cascio VM, Fu Y, Ho QH, Joung JK. CRISPR RNA-guided activation of endogenous human genes. Nat Methods. 2013 Oct $25 ; 10(10): 977-9$. 